\title{
Sensitive measurement of forces at micron scale using Bloch oscillations of ultracold atoms
}

\author{
I. Carusotto, ${ }^{1}$ L. Pitaevskii, ${ }^{1}$ S. Stringari,${ }^{1,2}$ G. Modugno, ${ }^{3}$ and M. Inguscio ${ }^{3}$ \\ ${ }^{1}$ Istituto Nazionale per Fisica per la Materia BEC-CRS and Dipartimento di Fisica, \\ Universitá di Trento, I-38050 Povo, Italy \\ ${ }^{2}$ École Normale Supérieure and Collège de France, \\ Laboratoire Kastler Brossel, 24 Rue Lhomond 75231 Paris, France \\ ${ }^{3}$ LENS and Dipartimento di Fisica, Università di Firenze, and INFM \\ Via Nello Carrara 1, 50019 Sesto Fiorentino, Italy
}

(Dated: November 9, 2018)

\begin{abstract}
We show that Bloch oscillations of ultracold fermionic atoms in the periodic potential of an optical lattice can be used for a sensitive measurement of forces at the micrometer length scale, e.g. in the vicinity of dielectric surface. In particular, the proposed approach allows to perform a local and direct measurement of the Casimir-Polder force which is, for realistic experimental parameters, as large as $10^{-4}$ gravity.

PACS numbers: $39.20 .+\mathrm{q}$, 03.75.Ss, 03.75.Lm
\end{abstract}

The study of forces at small length scales constitutes one of the challenges of current frontier research in physics. The interest spans from fundamental issues, like the search of possible deviations from newtonian gravity, to the phenomenology of forces close to surfaces. Accurate investigations have been performed using a variety of methods, as for instance the techniques based on microcantilevers 1, 2]. The laser production and manipulation of micron-size ultracold atomic samples has provided a new tool for the investigation of forces at small length scales [3, 4]. More recently, also trapped atomic BoseEinstein condensates have been applied to the study of forces close to surfaces [ 5, 6, 7].

The possibilities offered by quantum degenerate gases have been enriched by the combination with periodic trapping potentials, as those produced by optical lattices. As early demonstrated [8, 9, 10], this combination allows to study the general phenomenon of Bloch oscillations [11. A key feature of this phenomenon is that the measurement of an oscillation frequency, which can be made very precise, can be turned into a direct measurement of a force. An important step in this direction has been the observation of temporally-resolved Bloch oscillations of a degenerate Fermi gas of atoms trapped in an optical lattice and subjected to gravity [12. This has shown the feasibility of a high sensitivity force sensor with spatial resolution of the order of few microns.

In this paper we explore the effect of a weak inhomogeneous force close to a surface, such as the Casimir-Polder force [13], on atomic Bloch oscillations (BO). We make a complete theoretical analysis for a Fermi gas trapped in an optical lattice in proximity of a dielectric surface, using realistic parameters from an existing experiment 12 . We discover that this atomic Bloch oscillator realizes a powerful sensor for the detection of forces at small length scales.

The physical system that we consider here consists of a sample of ultracold atoms trapped in a 1D lattice aligned along gravity, as shown in Fig.1] The atoms are initially

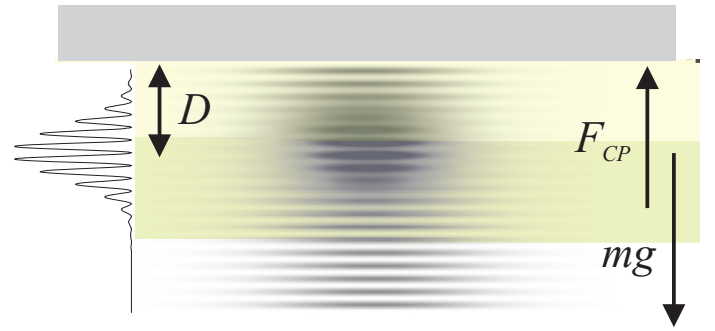

FIG. 1: Sketch of the physical situation considered in this paper: an ultracold sample of fermionic atoms is trapped in a vertical optical lattice in proximity of a dielectric surface and performs Bloch oscillations under the combination of gravity and of the Casimir-Polder force.

cooled in a harmonic potential, and then transferred to the optical lattice. A harmonic horizontal confinement is assumed to be present, e.g. given by the same reddetuned lattice beams. As soon as the vertical harmonic confinement is switched off, the atoms start to perform $\mathrm{BO}$ in the lattice potential under the action of gravity. If now a surface is brought close to the atomic sample, additional forces between the atoms and the surface will affect the dynamics of BO. We will now investigate how such forces can be measured from the shift of the Bloch oscillation frequency as the distance between atoms and surface is varied.

In particular, we consider here the Casimir-Polder force, which is exerted on an atom by the modified fluctuations of the electromagnetic field in the vicinity of a dielectric surface 13. As a first step, we have worked out the simplified model in which the Casimir-Polder (CP) force $F_{C P}$ is assumed to be spatially homogeneous. This is equivalent to assuming that the size of the sample along the vertical direction is small compared to the actual spatial variation of the force. In this case the quasimomentum $q$ of the atoms simply evolves as

$$
\hbar \dot{q}=F=m g+F_{C P}
$$


$F$ being the total force acting on the atom. As $q$ is defined only modulo the Bragg momentum $q_{B}=2 \pi / \lambda(\lambda$ is the wavelength of the optical lattice), each time $q$ gets to the edge of the first Brillouin zone at $q_{B}$, it reappears on the other side at $-q_{B}$. The period of $\mathrm{BO}$ is thus $T_{B}=4 \pi \hbar / F \lambda$. Following the evolution of BO for many periods one can infer their frequency, and therefore the Casimir-Polder force, with high accuracy. The fact that the frequency shift here is proportional to the force and not to its spatial gradient is an important difference of the present set-up with respect to previous work [7], and opens new possibilities for the measurement e.g. of radiation pressure forces close to a heated surface [14]. In 7] the effect of the $\mathrm{CP}$ force was investigated by looking at the shifts of the collective oscillation frequencies of a Bose-Einstein condensate confined in a harmonic trap. In this case the shift is proportional to the gradient of the force. Experiments in this direction are being performed by the group of E. Cornell at JILA [15].
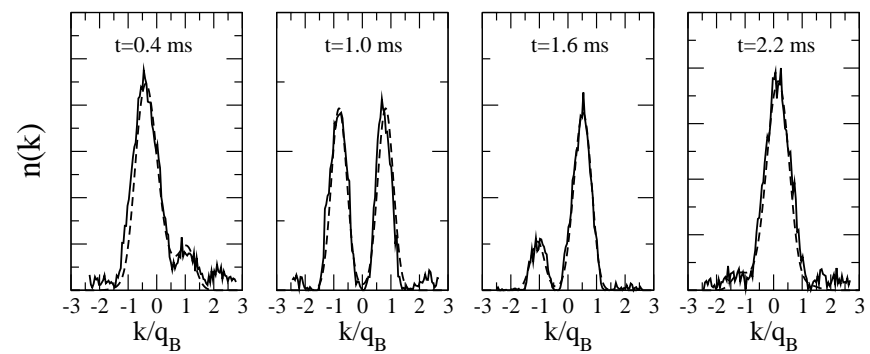

FIG. 2: Momentum distribution of a Fermi gas performing Bloch oscillations at different times within an oscillations period: solid lines are experimental data, dashed lines are theoretical calculations including adiabatic switch-off of the lattice. $E_{F} / 2 \delta=0.9$.

To observe BO one can study the momentum distribution of the atomic sample after a ballistic expansion. The typical evolution of the momentum is the one depicted in Fig.2. At the initial time $t=0$ the momentum distribution is centered at $k=0$, with symmetric lateral peaks at multiples of $2 q_{B}[16]$. As time goes on, the comb of peaks moves rigidly with constant velocity, while their relative intensity follows the Fourier transform of a Wannier state [17] as an envelope. In particular, when $q(t)$ reaches the edge of the first Brillouin zone at $t=T_{B} / 2$, the distribution contains a pair of symmetric peaks at $\pm q_{B}$. At $t=T_{B}$, the distribution has regained the initial shape. Note that the dynamics of BO in momentum space, being governed by the evolution equation (11) for the quasimomentum, is independent of the lattice height, while the corresponding oscillations in real space have an amplitude of order $\delta / F$, where $2 \delta$ is the width of the first energy band of the lattice. The presence of a strong known force, such as gravity, is therefore necessary to reduce the amplitude of the oscillation and consequently to achieve a high spatial resolution in the measurement of a weak force. The use of a vertical geometry is helpful also to prevent the atoms from hitting the surface during the ballistic expansion.

This discussion, originally developed for a single particle, is immediately extended to the case of a many-atom gas simply by convolving the single particle prediction by the initial quasi-momentum distribution of the gas: a fundamental requirement to clearly follow $\mathrm{BO}$ is that the quasi-momentum spread of the sample must not be much larger than $q_{B}$. This implies that the atomic temperature must be at most of the order of $2 \delta$. As the system size is limited by the spatial resolution we intend to obtain, a high density sample has to be used to increase the number of atoms and thus improve the signal. This motivates the use of a quantum degenerate gas.

Although a Bose-Einstein condensate would certainly offer a much narrower initial quasi-momentum spread than a Fermi gas, it has been shown in recent experiments 18 to suffer from instabilities due to interactions, which quickly broaden the quasi-momentum distribution and make the observation of $\mathrm{BO}$ practically impossible in condensates at high density. As a matter of fact, for the rest of the paper we will mainly consider a Fermi gas: thanks to the absence of interactions this appears to be an excellent candidate for this kind of experiments [12]. For simplicity we will consider a Fermi gas at $T=0$, whose quasi-momentum distribution is:

$$
N_{q}=\frac{1}{2}\left(\frac{E_{F}-E_{q}}{\hbar \omega_{r}}\right)^{2} \Theta\left(E_{F}-E_{q}\right)
$$

where $E_{q}$ is the energy of the Bloch state of quasimomentum $q, E_{F}$ is the Fermi energy, and $\omega_{r}$ is the frequency of the radial harmonic trapping. As long as $E_{F}$ is smaller than $2 \delta$, the quasi-momentum distribution is localized in a region around $q=0$ and is zero outside, while the contrast slowly deteriorates for larger values of $E_{F}$. Notice that this behavior is different from the case of a purely one-dimensional system, where the contrast would be strictly zero as soon as $E_{F} \geq 2 \delta$ [19.

The contrast can be improved by adiabatically switching off the lattice potential at the end of the BO. In this case, the population of the state of quasimomentum $q$ is projected onto the first Brillouin zone without being weighted by the Fourier transform of the Wannier state and at most two peaks are observed. In Fig.2] we report the result of a theoretical analysis taking into account the adiabatic switch-off of the lattice, which reproduces with excellent accuracy the experimental data taken as described in 12.

Let us now study the effect of the $\mathrm{CP}$ force between the atoms and the dielectric surface. A general discussion of its properties has been recently given by [7], and we refer to that paper for the details of the calculations. In the simulations for the present paper, the potential plotted in Fig. 3 of [7] has been used, which takes into account all the relevant regimes (van der Waals-London, CasimirPolder, and thermal), in particular the thermal one

$$
V_{C P}^{(t h)}(z)=-\frac{k_{B} T \alpha_{0}}{4 z^{3}} \frac{\epsilon_{0}-1}{\epsilon_{0}+1}
$$




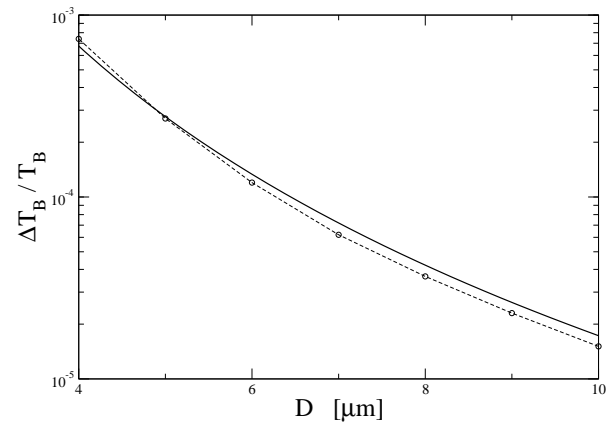

FIG. 3: Frequency shift of Bloch oscillations due to CasimirPolder corce. The solid line is the prediction of Eq4 4 based on the thermal potential (3), circles are the result of a complete numerical simulation as described in the text.

which dominates at distances larger than the thermal wavelength $\hbar c / k_{B} T$ of the photon. Here $\alpha_{0}$ is the static atomic polarizability, $\epsilon_{0}$ is the static dielectric constant of the material composing the surface, which is placed at $z=0$, the $z$ axis being oriented downwards. At the lowest level, one can approximate the spatially varying force with a homogeneous one equal in magnitude to the value of thermal force (3) evaluated at the center of the cloud. In this case, the BO period for an atomic cloud at a distance $D$ from the surface is easily evaluated from eq1 and has a relative shift

$$
\frac{\Delta T_{B}}{T_{B}}=-\frac{F_{C P}(D)}{m g}=\frac{0.1728}{D^{4}}(\mu \mathrm{m})^{4} .
$$

The numerical value refers to the specific case of potassium atoms and a sapphire surface with $\epsilon_{0}=9.4$ and $T=300 \mathrm{~K}$. In Fig. 3 we show the relative BO period shift as a function of the distance $D$, computed according to the approximated formula (4). Note how the effect of the Casimir potential ranges from $10^{-4}$ to $10^{-5}$ at distances $D$ from 5 to 10 microns, which are realistic for a possible experiment. This situation is encouraging because such level of sensitivity has been already demonstrated in a proof-of-principle experiment [12], and can be further improved by a few orders of magnitude, as we will discuss later.

It is then worth performing a deeper analysis taking fully into account the spatial inhomogeneity of the Casimir force over the atomic cloud, its full $z$ dependence beyond the large distance approximation (3) as well as the effect of a real-space motion of the cloud during BO. We have indeed performed an exact numerical calculation for the non-interacting many fermion problem, in the combined potential of the trap, the gravity, the lattice and the complete $\mathrm{CP}$ force as predicted in [7]. This has been done by first obtaining the single-particle orbitals in the initial potential of the trap, the lattice, and the $\mathrm{CP}$ force, by then populating them according to a Fermi distribution, and by finally propagating them via the Schrödinger equation in the combined potential of the lattice, the gravity and the $\mathrm{CP}$ force. This proce- dure provides the density profile of the gas, as well as its momentum and quasi-momentum distributions at all times. From the center-of-mass of the quasi-momentum distribution, it is then possible to extract a theoretical prediction for the $\mathrm{BO}$ frequency shift.
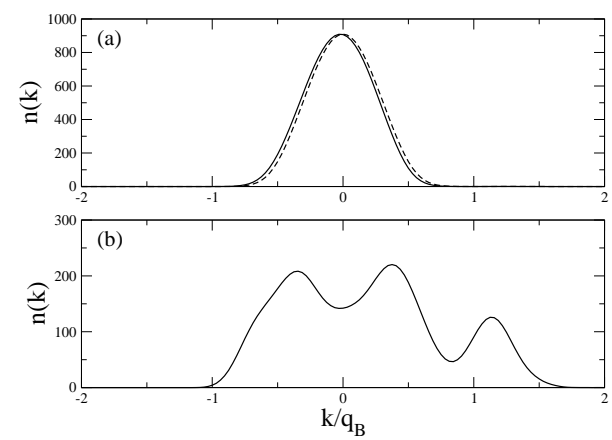

FIG. 4: Upper panel (a): momentum distribution of a Fermi gas at a distance $D=10 \mu \mathrm{m}$ from the surface at beginning (dashed) and after 1000 periods of Bloch oscillations (solid). Lower panel (b): momentum distribution of a BEC after 2 periods of Bloch oscillations.

The results of such a calculation are shown in Fig. 3 for realistic parameters: a situation has been considered in which $2 \cdot 10^{4}{ }^{40} \mathrm{~K}$ atoms are prepared in a lattice with $\lambda=873 \mathrm{~nm}$, with a vertical extension of about $4 \mu \mathrm{m}$, i.e. 8 lattice sites. To achieve a favourable ratio $E_{F} / 2 \delta=0.9$ close to one we have chosen a lattice height $s=5$, and a radial frequency of $10 \mathrm{~Hz}$. The vertical extension in the lattice is determined by the confinement of the initial harmonic potential of a trap, which for this specific case has a frequency of $400 \mathrm{~Hz}$. Justification for the slight difference with respect to the analytical formula (4) can be found in several effects that have been neglected in the previous discussion, in particular the spatial displacement (on the order of $1 \mu \mathrm{m}$ in the direction of going further from the surface) of the atomic cloud while performing $\mathrm{BO}$, the difference between the approximate thermal potential (3) and the exact one of [] and finally the spatial inhomogeneity of the $\mathrm{CP}$ force across the atomic cloud. The inhomogeneity of the force can have a further consequence: the frequency shift of $\mathrm{BO}$ is in fact given by an average of the force over the cloud profile, and a sort of inhomogeneous broadening is to be expected. As this latter effect may affect the accuracy of the measurement, in particular after the large number of oscillations which are required for a high-precision measurement, we have investigated the broadening of the momentum distribution after a large number of $\mathrm{BO}$ periods. The results, reported in Fig. 4 , show that the broadening is not yet significant after 1000 periods.

For the sake of completeness, a complete analysis has been also performed for the bosonic case by numerically solving the 1D Gross-Pitaevskii equation with trap and atom number parameters analogous to the Fermi case (that is with a peak density of $n=4 \cdot 10^{13} \mathrm{~cm}^{-3}$ ). The results are in agreement with the experimental obser- 
vations of [12]: the quasi-momentum distribution dramatically broadens as soon as the quasi-momentum of the Bose condensate enters the dynamically instable region [20] and after only a few BO periods, it is not possible to follow the oscillations any longer (Fig.4 $4 \mathrm{~b}$ ). To overcome this problem, one could think of setting the scattering length $a$ to zero by means of a Feshbach resonance [21]. As the characteristic time for the onset of the instability is of the order of $\tau_{\text {inst }} \approx \hbar / g n_{\max }[20$, $g n_{\max }=4 \pi \hbar^{2} a n_{\max } / m$ being the mean-field interaction energy, an upper bound on the absolute value of $a$ is set by the fact that $\tau_{\text {inst }}$ has to be much longer than the actual duration of the measurement. For instance, a measurement lasting $100 \mathrm{BO}$ periods, performed with the atomic density here considered, would require a reduction of the scattering length of a factor of the order of $10^{3}$.

Let us now discuss the sensitivity that can be realistically achieved in an experiment. In [12] we demonstrated an overall sensitivity $\Delta T_{B} / T_{B}=10^{-4}$, by following about $100 \mathrm{BO}$ periods of ${ }^{40} \mathrm{~K}$ atoms in a lattice with $\lambda=873 \mathrm{~nm}$. This sensitivity was limited by various sources of decoherence due to the imperfect nature of the optical lattice. Examples are the phase noise due to mechanical vibrations of the retroreflecting mirror, or longitudinal inhomogeneous forces due to the focusing of the lattice beams. Most of these decoherence sources can be reduced in an optimized experiment, thus extending the number of oscillations observable with high contrast. The typical lifetime of the atomic sample of a few seconds will however limit the realistic number of observable $\mathrm{BO}$ periods to the order of $10^{3}$. Since the sensitivity on the measurement of a single $\mathrm{BO}$ period can be pushed to $10^{-3} \div 10^{-4}$, as typically achieved in this kind of experiments 22], one can expect to obtain an overall sensitivity $\Delta T_{B} / T_{B}=10^{-6} \div 10^{-7}$.

This sensitivity would certainly allow to study a weak and inhomogeneous force like the Casimir-Polder force already at distances larger than $5 \mu \mathrm{m}$ from a dielectric surface. This level of accuracy might also open to the investigation of possible deviations from Newton's gravitational law at short distance 23]. In order to improve the probing of the force at shorter distances one should reduce the width of the sample by working, for example, with a stronger vertical confinement and with a shorter lattice periodicity. The latter would indeed work in favor of a higher resolution, without affecting the sensitivity because, as already discussed, the measurable quantity $\dot{q}$ depends only on the applied force.

In conclusion we have discussed how Bloch oscillations can provide a sensitive tool for the study of forces at the micrometer scale, e.g. the Casimir-Polder force between atoms and dielectric surfaces. The frequency shift of Bloch oscillations is proportional to the applied force and at a distance of the order of a few microns turns out to be well within the sensitivity of realistic experiments.

We are grateful to M. Antezza for continuous stimulating discussions on the Casimir-Polder forces. Discussions with G. Roati, F. Ferlaino, G. Orso, C. Tozzo and F. Dalfovo are also acknowledged. M.I. aknowledges the support of the Institute d'Optique, Orsay, and the stimulating hospitality in the group af A.Aspect. This work was supported by MIUR and by EU under contract HPRICT1999-00111.
[1] J. Chiaverini, S. J. Smullin, A. A. Geraci, D. M. Weld, and A. Kapitulnik, Phys. Rev. Lett. 90, 151101 (2003).

[2] G. Bressi, G. Carugno, R. Onofrio, and G. Ruoso, Phys. Rev. Lett. 88, 041804 (2002).

[3] C. I. Sukenik, M. G. Boshier, D. Cho, V. Sandoghdar, and E. A. Hinds, Phys. Rev. Lett. 70, 560 (1993).

[4] F. Shimizu, Phys. Rev. Lett. 86, 987 (2001).

[5] Y. Lin, I. Teper, C. Chin, and V. Vuletić, Phys. Rev. Lett. 926, 050404 (2004).

[6] J. M. McGuirk, D. M. Harber, J. M. Obrecht, and E. A. Cornell, Phys. Rev. A 69, 062905 (2004).

[7] M. Antezza, L. P. Pitaevskii, and S. Stringari, Phys. Rev. A 70, 053619 (2004).

[8] M. Ben Dahan, E. Peik, J. Reichel, Y. Castin, and C. Salomon, Phys. Rev. Lett. 76, 4508 (1996).

[9] S. R. Wilkinson, C. F. Bharucha, K. W. Madison, Q. Niu, and M. G. Raizen, Phys. Rev. Lett. 764512 (1996).

[10] B. P. Anderson and M. A. Kasevich, Science 282, 1686 (1998).

[11] C. Zener, Proc. R. Soc. London Ser. A 145, 523 (1934).

[12] G. Roati, E. de Mirandes, F. Ferlaino, H. Ott, G. Modugno, and M. Inguscio, Phys. Rev. Lett. 92, 230402 (2004).

[13] H.B.G. Casimir and D. Polder, Phys. Rev. 73, 360 (1948).
[14] C. Henkel, K. Joulain, J.-P. Mulet and J.-J. Greffet, J. Opt. A: Pure Appl. Opt. 4, S109 (2002).

[15] E. Cornell, D. Harber and J. Obrecht, private communication.

[16] P. Pedri, L. Pitaevskii, S. Stringari, C. Fort, S. Burger, F. S. Cataliotti, P. Maddaloni, F. Minardi, and M. Inguscio, Phys. Rev. Lett. 87, 220401 (2001)

[17] P. Y. Yu and M. Cardona, Fundamentals of semiconductors, Springer, Berlin, 1996.

[18] L. Fallani, L. De Sarlo, J. E. Lye, M. Modugno, R. Saers, C. Fort, and M. Inguscio, Phys. Rev. Lett. 93, 140406 (2004).

[19] L.Pezzé, L.Pitaevskii, A. Smerzi, S.Stringari, G. Modugno, E. De Mirandes, F. Ferlaino, H. Ott, G. Roati, M. Inguscio, Phys. Rev. Lett. 93, 120401 (2004).

[20] B. Wu and Q. Niu, Phys. Rev. A 64, 061603 (2001).

[21] T. Weber, J. Herbig, M. Mark, H.-C. Nägerl, R. Grimm, Science 299, 232 (2003).

[22] R. Battesti, P. Cladé, S. Guellati-Khélifa, C. Schwob, B. Grémaud, F. Nez, L. Julien, and F. Biraben Phys. Rev. Lett. 92, 253001 (2004).

[23] S. Dimopoulos and A. A. Geraci Phys. Rev. D 68, 124021 (2003). 